\title{
HIGHLY IONIZED ATOMS IN TOKAMAK DISCHARGES
}

\section{PLASMA PHYSICS LABORATORY}

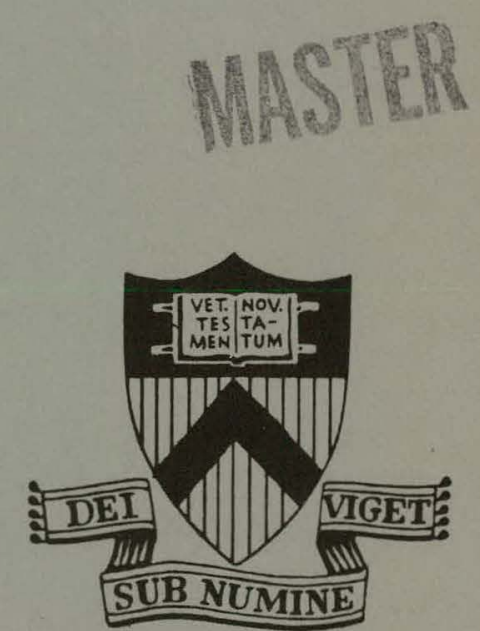

\section{PRINCETON UNIVERSITY PRINCETON, NEW JERSEY}

This work was supported by U. S. Energy Research and Development Administration Contract E(11-1)-3073. Reproduction, translation, publication, use and disposal, in whole or in part, by or for the United States Government is permitted. 


\section{DISCLAIMER}

This report was prepared as an account of work sponsored by an agency of the United States Government. Neither the United States Government nor any agency Thereof, nor any of their employees, makes any warranty, express or implied, or assumes any legal liability or responsibility for the accuracy, completeness, or usefulness of any information, apparatus, product, or process disclosed, or represents that its use would not infringe privately owned rights. Reference herein to any specific commercial product, process, or service by trade name, trademark, manufacturer, or otherwise does not necessarily constitute or imply its endorsement, recommendation, or favoring by the United States Government or any agency thereof. The views and opinions of authors expressed herein do not necessarily state or reflect those of the United States Government or any agency thereof. 


\section{DISCLAIMER}

Portions of this document may be illegible in electronic image products. Images are produced from the best available original document. 
This report was prepared as an account of work sponsorcd by the United States Government. Neither the United States nor the United States Energy Research and Development Administration, nor any of their employees, nor any of their contractors, subcontractors, or their employees, makes any warranty, express or implied, or assumes any legal liability or responsibility for the accuracy, completeness or usefulness of any information, apparatus, product or process disclosed, or represents that its use would not infringe privately owned rights.

Printed in the United States of America.

Available from

National Technical Information Service

U. S. Department of Commerce 5285 Port Royal Road

Springfield, Virginia 22151

Price: Printed Copy \$_*; Microfiche $\$ 1.45$.

NTIS

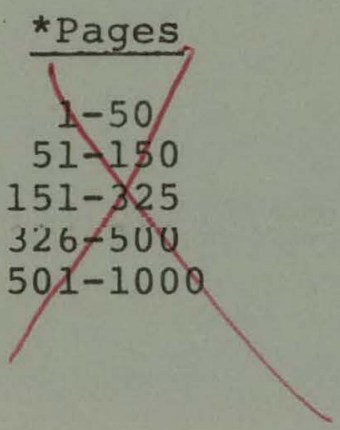

Selling Price

$\$ 4.00$

5.45

7.60

$10 \% 60$

13.60 


\title{
HIGHLY IONIZED ATOMS IN TOKAMAK DISCHARGES
}

\author{
E. Hinnov
}

Plasma Physics Laboratory, Princeton University Princeton, New Jersey 08540

Tokamak discharges are characterized by electron densities usually $\sim 0.3-1.0 \times 10^{14} \mathrm{~cm}^{-3}$ and temperatures from a few hundred $\mathrm{eV}$ to several $\mathrm{keV}$. In addition to the working gas ( $\mathrm{H}$ or $\mathrm{He}$ ), the plasma normally contains some light impurities $\left(\sim 10^{12} \mathrm{~cm}^{-3} 0\right.$ or $\left.\mathrm{C}\right)$ that are completely stripped except at the outer periphery, and heavier elements from the vacuum wall and current-aperture limiter (Fe, $\mathrm{Cr}, \mathrm{Ni}, \mathrm{W}$, Mo and others, $2.0^{10}-10^{\mathrm{il}} \mathrm{cm}^{-3}$ ) that remain partly stripped, hence relatively strongly radiating, throughout the discharge. Other elements, especialiy noble gases, may be deliberately added for diagnostic purposes. Resonance lines of Fe and $\mathrm{Ar}$ in the beryllium and lithium sequences, of $\mathrm{Fe}, \mathrm{Kr}$, and Mo in the magnesium and sodium sequences, and of Mo and Xe in the zinc and copper sequences have been used for rough deter... mination of plasma composition. Since crucial plasma characteristics such as temperature and confinement time are sensitively affected by the local composition, it is essential to improve the available atomic data necessary for more accurate analysis: wavelengths, transition probabilities, excitation, ionization and recombination rates, especially for the heavier elements.

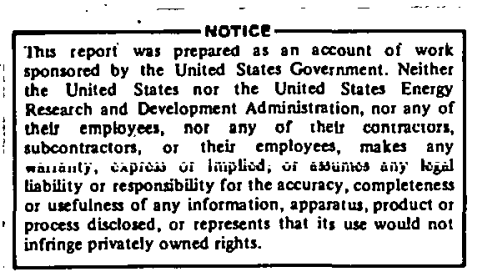




\section{INTRODUCTION}

The tokamak discharge produces plasmas that can vary over a considerable range in electron temperature, $\mathrm{T}_{\mathrm{e}}$, both in space and time in.a given discharge, and in discharges of different initial or boundary conditions. For the purpose of producing light of highly ionized atoms, it is the highest central temperatures that are of primary interest. These vary in present-day tokamaks from about $1.5-3, \mathrm{keV}$, i.e. roughly comparable to temperatures reached in solar flares. The other parameter of interest in producing highly ionized atoms is the product of electron density $\mathrm{n}_{e}$ and the ion confinement time $\tau$, which reaches values of $\mathrm{n}_{e^{\tau}} \approx 1-3 \times 10^{12} \mathrm{sec} / \mathrm{cm}^{3}$ in the discharges described in the present paper. The highest state of ionization reached by a given atom is determined either by this product, or the inverse rate coefficient of recombination at the ambient temperature, whichever is smaller. Generally, plas-: mas of highest temperature also have the longest confinement times, but the study of highly ionized atoms is complicated by the fact that their preserice in sufficient quantities to be observable also affects adversely the achieved temperatures and confinement times.

The working gas, that provides the bulk of the plasma is usually hydrogen or deuterium, sometimes helium. The plasma generally contains several percent of oxygen and lesser amounts of carbon desorbed from the walls. These affect the plasma behavior considerably, but they are not of direct concern in the present paper. The heavy atoms under discussion are 1) those of stainless steel' vacuum walls, and molybdenum current-aperture limiters, that are released by sputtering or evaporation during the discharge, 
and 2) atoms of noble gases deliberately introduced in controlled quantities for diagnostic purposes. In this paper we report the measurement of wavelengths and intensitles, and the identification of the electronic transitions, of several resonance lines of highly ionized states of these atoms.

\section{EXPERIMENTAL SETUP}

The light source used in the present work was the Princeton ST Tokamak. Since the operation of tokamaks generally, and also of the ST tokamak ${ }^{2-4}$ have been amply discussed in recent literature, we shall confine the description of the discharge only to such features that are directly relevant to the observation of the highly ionized ion resonance lines.

The plasma is produced in a toroidal magnetic field, within a stainless steel vacuum vessel of $15.8 \mathrm{~cm}$ minor and $109 \mathrm{~cm}$ major radius by a toroidal current $I_{\mathrm{OH}^{\prime}}$ in an initial working gas $\mathbf{( H}_{2}$ or He) density of about $10^{13} \mathrm{~cm}^{-3}$. At one toroidal position a heavy rectangular (usually $12 \times 13 \mathrm{~cm}$ ) molybdenum aperture limits the current dimensions and reduces the interaction between hot plasma and the vacuum vessel.

Typical behavior of a discharge is shown in Fig. 1. The heating current $I_{O H}$ rises within a few milliseconds to about 60 kiloamperes, and thereafter increases slowly to a flat maximum of about 80 kamps some 60-70 msec. later. The average electron density (number per $\mathrm{cm}^{2}$ along the line of sight in the equatorial plane of the torus, divided by limiter aperture) rises very rapidly to an initial peak, and varies slowly thereafter, either rising slowly as in Fig. l or perhaps falling slowly under some other 
discharge conditions. The electron density behavior is determined by approximate balance between particle loss by diffusion etc., and continued ionization of the recycled particles i.e. those neutralized on the walls or the limiter returning to the plasma. The average confinement time, i.e., the characteristic time a given atom stays in the plasma is only a few milliseconds in the early phase and perhaps 15-20 msec in the quasisteady part of the discharge. The confinement time appears to be at least approximately the same for all plasma constituents: electrons, protons and the various impurity ions.

The insets in Fig. 1 describe the radial distributions of electron density and temperature at different times during the discharge. In the early phase, $\sim 5 \mathrm{milliseconds}$ on the scale of the main figure, the electron density is usually fairly uniform, although various fine structures may occur under some conditions. The electron temperature may have risen to 300-400 ev , and often shows a radial shell structure as indicated, caused by the current skin-effect. About $10 \mathrm{msec}$ later the density profile has become approximately parabolic, which shape with perhaps minor modifications persists throughout the rest of the discharge. The electron temperature distribution at around $15 \mathrm{msec}$ has developed a peaked profile with a height of perhaps $2 \mathrm{keV}$ or more, and the peak subsequently broadens to a flat plateau, with a width of $6-8 \mathrm{~cm}$, but the profile remains considerably narrower than the density profile, i.e. there is a hot plasma core surrounded by a considerably cooler plasma of similar density. An alternative temperature profile development is depicted by the dotted curves: here the central tempenature fails to grow beyond about $1.5 \mathrm{kev}$, and 
although it may broaden somewhat in time; tends to retain a roughly triangular shape. This latter distribution development is associated with higher concentrations of heavy impurities, either wall materials or added elements. A high state of ionization of a given element may thus be unobservable either because there is too little of the element present, or too much, and the conditions of optimum observability are often difficult to establish.

The resonance lines of the first five states of oxygen appear successively during the first few milliseconds of the discharge, go through pronounced maxima during the time indicated on Fig. 1 (some samples are presented below) and subside to quasisteady plateaux of intensity that originate from the outer periphery of the plasma, where the electron temperature is comparable to the ionization potential of the ion on question, i.e. $\sim 100 \mathrm{ev}$. From about 7-12 msec appear qualitatively similar lines of ions of ionization potential of several hundred eV, such as Mo XIII and XIV, Fe XV and XVI, O VII, and others. Finally, at times of $230 \mathrm{msec}$ and later, there appear the lines of the highly ionized atoms that constitute the main topic of this paper.

The line intensities were measured with a Spex lm vacuum monochromator equipped with a Jobin-Yvon holographically ruled goldcoated 1200 groove/mm grating at 830 angle of incidence, and Bendix magnetic electron multiplier with tungsten cathode. The instrumental bandwidth was approximately $1 \AA$, determined by the width of the slits. The quoted wavelengths are accurate to $\pm 0.5 \AA$ in the case of stronger lines that could be clearly distinguished from continuum emission and neighboring lines and scattered light, principally due to oxygen. The instrument was calibrated in situ by means of the line-ratio 
method $^{5}$, with results shown in Fig. 2. Each of the measured points is reproducible to about 208 or better. The points represented by neutral helium lines are only slightly affected by selfabsorption because at the time of measurement the emission line is much wider than the absorption line, due to resonant chargeexchange collisions in the plasma ${ }^{6}$. The points at $L_{\ddot{\alpha}}$ and HeII 256-304A are based on calculated relative populations of the $\mathrm{n}=2,3$, and 4 levels. ${ }^{7}$. These calculations are probably better than the measurement accuracy, except for the case of the $\lambda 304 \stackrel{\circ}{\AA}$ line, where the indicated spread is due to the uncertainty of electron temperature at the time of measurement. The interpolated curve is then used to measure the unknown line intensities. Fortunately, most of the lines discussed in this paper are not very far from measured points.

The intensities are measured as surface brightness, number of photons per sec, $\mathrm{cm}^{2}$ and steradian, in the midplane of the torus. At the time of peak intensity, the local emissivity is given by multiplying the brightness by $4 \pi / \Delta r$, with $\Delta \mathrm{r} \approx 20-24$ cm for the early states of ionization, during the first several milliseconds of the discharge, where $n_{e}(r)$ and $T_{e}(r)$ are fairly flat, and $\Delta r \because 6-8 \mathrm{~cm}$ for the higher states of ionization, corresponding to the narrow high-temperature part of the discharge. The drop of the intensity after the maximum, while the electron density and temperature are steady or increasing, then indicates decrease of the effective $\Delta r$, usually by ionization to the next state near the center of the plasma and the consequent development of the cylindrical shell 
structure of the radiation mentioned above.

\section{RESULTS}

The deliberately added elements such as the noble gases provide important advantages in identification of the lines: the element is a priori known, and the amount can be adjusted for optimum observation conditions. We therefore discuss these cases first.

a) krypton. The data obtained in a discharge with an initial gas filling of deuterium $\left(6.6 \times 1.0^{12} \mathrm{D}_{2} \mathrm{~mol} . / \mathrm{cm}^{3}\right)$ with $2.9 \times 10^{10}$ atoms $/ \mathrm{cm}^{3}$ of krypton are given in Fig. 3, and the early part of the discharge with expanded time and contracted intensity scales in Fig. 4 .

The early electron density is roughly double that of the initial

D atom density. This is almost entirely due to the ratio of the vacuum vessel volume to plasma volume. The contribution of other constituents, even of oxygen is small at this time, and remains rather modest even at later times. The variation of $\bar{n}_{e}$ in time is determined practically entirely by plasma confinement and recycling and thus only indirectly (through electron temperature, electrical resistivity etc.) through composition.

The central temperature rises nearly linearly to $1.5 \mathrm{keV}$ and thereafter levels off. However, from about 60-70 msec there is a somewhat unusual additional rise to about $1.9 \mathrm{keV}$, which may be associated with a substantial part of the krypton in the center reaching the neon-like configuration and thereby decreasing its radiation efficiency.

In discussing the radiation, we note that in the case of all the spectrum lines mentioned in this paper, the lines are emitted 
from a plasma at an electron temperature such that

$$
k \mathrm{~T}_{\mathrm{e}} \approx \mathrm{E}_{\mathrm{i}} \gg \mathrm{E}_{\mathrm{x}}
$$

where $E_{i}$ is the ionization potential of the ion in question, and $E_{x}$ is the excitation potential for the line. Furthermore, the various relevant transition rates are such that the line emission is practically exclusively due to electron collisional transitions from the ground state of the ion (lower state of the transition), followed by spontaneous radiative de-excitation. Under these conditions the line intensity is very nearly independent of $\mathrm{T}_{\mathrm{e}^{\prime}}$ and also depends very weakly on the near-threshold excitation cross-sections or effective Gaunt factors ${ }^{8-10}$, and scales as

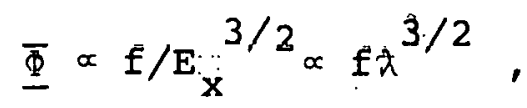

with $f$ the absorption oscillator strength and $\lambda$ the wavelength for the line. The observed intensity variations are then due to the variation of the particular state of ionization of the atom (along the line of sight), and the nearly constant electron density.

Kr VII and Kr VIII lines peak at a time when the electron temperature has reached about $100-130 \mathrm{ev}$, (comparable to the ionization potential $111 \mathrm{eV}$ and $123 \mathrm{eV}$, respectively). The oxygen ions $\mathrm{OV}\left(\mathrm{E}_{\mathrm{i}}=114 \mathrm{eV}\right)$ OVI $\left(E_{i}=138 \mathrm{eV}\right)$ peak slightly later. The delay may be due to initial conditions: oxygen is injected mostly as desorbed water vapor during the first millisecond or so of the discharge, whereas krypton presumably occupies the volume from the start. An interesting feature is the inversion of the relative intensities: the $2 s^{2}-2 s 2 p$ 
singlet line of $\mathrm{ov}$ is weaker than the $2 \mathrm{~s}^{2} \mathrm{~s}_{1 / 2}-2 \mathrm{p}{ }^{2} \mathrm{P}_{3 / 2}$ ine of OVI (almost exactly the same relationship has been recently observed in the Tokamak of the Fontenay-aux-Roses ${ }^{11}$, , whereas the reverse is true of the $n=4$ transitions in krypton. Aside from experimental uncertainties, there are two probable causes for this phenomenon. According to the best available theoretical cross-section, the rate coefficients for excitation ${ }^{11}$ of the ov and OVI lines at about $100 \mathrm{eV}$ electron temperature are $2.8 \times 10^{-8} \mathrm{~cm}^{3} / \mathrm{sec}$ and $1.7 \times 10^{-8} \mathrm{~cm}^{3} / \mathrm{sec}$ respectively, whereas an adaption of the same procedure of calculation for the Kr VII and Kr VIII lines (also used for other lines quoted below) gives $5.5 \times 10^{-8}$ and $2.4 \times 10^{-8} \mathrm{~cm}^{3} / \mathrm{sec}$ respectively. Another and perhaps more important question in the intensity inversion is the relative importance of the triplet $\mathrm{nsinp}{ }^{3} \mathrm{P}$ populations relative to the singlet ground state. It appears from a: comparison of singlet and triplet line intensities of ov that about half or slightly more of the ov population is in the triplet state at the electron densities and temperatures in question, which incidentally explains why the ov line is weaker than the OVI line with its smaller excitation rate coefficient. Corresponding information about the triplet in $\mathrm{Kr}$ VII is unfortunately not available, but the intensity relationship with $\mathrm{Kr}$ VIII suggests that the proportion of triplets here is relatively less. This, and analogous problems in other elements particularly those with $\mathrm{ns}^{2}$ ground configuration evidently need further investigation.

Another possible explanation of the intensity changes would be a rapid increase of the relative oxygen concentration or decrease of 
krypton concentration in time. However, other evidence, including the subsequent behavior of the discharge 2,12 , and the soft $x$-ray continuum and krypton $\mathrm{K}-1$ ine intensity ${ }^{4}$, indicates that this is not the case - the relative concentrations change only very slowly, if at all, on the time scale of the discharge.

The $\lambda 284 \AA, F_{e} X V\left(E_{i}=456 \mathrm{eV}\right)$ line appears at a time when the central electron temperature exceeds $300 \mathrm{eV}$, as expected from the rates of ionization. The excitation rate coefficient for this line is about $1 \times 10^{-8} \mathrm{~cm}^{3} / \mathrm{sec}$.

Similar calculations for the appearance of the magnesium-like $\operatorname{Kr} \operatorname{XXV}\left(E_{i}=1151 \mathrm{eV}\right)$ and sodiumlike $\operatorname{Kr} \operatorname{XXVI}\left(E_{i}=1206 \mathrm{eV}\right)$ indicate that they should appear between 20 and $30 \mathrm{msec}$ on the time-scale of Fig. 3. The approximate location of the resonance lines of these ions were extrapolated from known lines of the isoelectronic sequences by a procedure described by Edién ${ }^{13}$, and a search in the neighborhood of the predicted lines yielded the three lines shown in Fig. 3. The limit of sensitivity was about a factor 2-3 below the intensity of the $221 \AA$ line, and within this limit no other krypton line of similar time behavior (i.e. of the same or nearby states of ionization) were found between $130-250 \AA$. We therefore have little doubt about their identification: $\lambda 159.0 \AA \mathrm{Kr} \mathrm{XXV} 3 \mathrm{~s}^{2}{ }^{1} \mathrm{~s}$ - $3 \mathrm{~s} 3 \mathrm{p}{ }^{1} \mathrm{p}$, and $\lambda \lambda 179.6,220.6 \AA \mathrm{Kr} \operatorname{xxVI~} 3 \mathrm{~s}^{2} \mathrm{~s}-3 \mathrm{p}^{2} \mathrm{P}_{3 / 2}{ }_{1 / 2}$ respectively. The predicted rate coefficients for excitation are $2.6 \times 10^{-9} \mathrm{~cm}^{3} / \mathrm{sec}$ for the $\lambda 159 \AA$ line, and $1.6 \times 10^{-9}$ for the sur of the other two, at $1.5 \mathrm{keV}$ temperature. These numbers are in reasonable agreement with the relative intensities, again considering that some of the Kr XXV population should be in the triplet state. The ratio of the $\mathrm{Kr}$ XXVI lines appears to vary in time from about 1.6 early to 2.0 late in the discharge, but this is probably 
due to difficulties in allowing for background radiation, which is in intensity comparable to the weaker line, especially in later part. According to the $f \lambda^{3 / 2}$ scaling, the expected ratio using the f-values of Weiss and wiese ${ }^{14}$ should be 1.8 , in reasonable accord with observation.

Compared to the earlier $\mathrm{Kr}$ VII and VIII lines, the $\mathrm{Kr} X X V, \mathrm{XXVI}$ line intensities are weaker by a factor 80-90. Most of this, a factor 20-25 is ascribed to the excitation rate coefficients quoted above, the rest, about a factor 4 , to the shortening of the pathlength, $\Delta r$, along the line of sight where the radiation originates, according to the temperature profiles explained in Fig. 1. In other words, the quoted rate coefficients for excitation are consistent with the assumption of no significant change in time of the relative proportion of krypton in the plasma composition.

b) Xenon. The appearance of the xenon lines, $\lambda 164.5 \AA$ ascribed to $4 \mathrm{~s}^{2}{ }^{1} \mathrm{~s}-4 \mathrm{~s}^{4} \mathrm{p}^{1} \mathrm{P}$ of $\mathrm{Xe} \mathrm{XXV}$, and $\lambda \lambda 173.9,234.2 \AA$ ascribed to $4 \mathrm{~s}^{2} \mathrm{~s}-4 \mathrm{p}^{2} \mathrm{P}_{3 / 2}, 1 / 2$ of Xe XXVI are shown in Fig. 5. The hydrogen pressure and heating current were about the same as in the krypton case, but the Xe initial pressure was higher, about $9 \times 10^{10}$ atoms $/ \mathrm{cm}^{3}$. This was sufficient to significantly depress the electron temperature, which reached only about $800 \mathrm{eV}$ at $20-30 \mathrm{msec}$. As a consequence of the lowered temperature the particle confinement time also was smaller, and resulted in lower electron density. Nevertheless, the Xe XXV and Xe XXVI ions are readily produced because of their relatively low ionization potentials (853 and $891 \mathrm{ev}$, respectively). The only qualitative difference from the krypton lines is the relatively higher intensity of the $\lambda 174 \AA$, ${ }^{2} \mathrm{~S}-{ }^{2} \mathrm{P}_{3 / 2}$ line with respect to both $\lambda 234 \AA$ and $\lambda 165 \AA$ ines. 
The copper sequence wavelengths are in good agreement with recent relativistic Hartree-Fock calculations $1173.4,233.7 \AA$ at Systems, science and software ${ }^{15}$ and $180.8,237.3 \AA$ at NBS ${ }^{16}$ ). The latter reference also includes calculation of oscillator strengths; according to which the line ratio should again be 1.8 , somewhat smaller than the measured ratio of about 2.1 .

c) Argon. An analogous experiment with $2.2 \times 10^{10} \mathrm{~cm}^{-3}$ argon added to hydrogen discharge yielded the following intensities and wavelengths at a time when $\bar{n}_{e}=1.4 \times 10^{13}, T_{e}(0)=1.2 \mathrm{keV}$. For $2 \mathrm{~s}^{2}-2 \mathrm{~s} 2 \mathrm{p}$ Ar XV $\left(\mathrm{E}_{\mathrm{i}}=855 \mathrm{ev}\right)$, intensity $5.5 \times 10^{14} / \mathrm{cm}^{2}-\mathrm{sec}-\mathrm{sr}$; $\lambda=221.2 \AA ;$ for $2 \mathrm{~s}-2 \mathrm{p}$ Ar XVI $\left(E_{i}^{\prime}=918 \mathrm{eV}\right)$, intensities 4.1 and $2.5 \times 10^{14}$ of $\lambda=354.1 \AA$ and $389.3 \AA$ 1ines, respectively. These 1.ines had been identified previously ${ }^{17,18}$, and their wavelengths agree with the published values well within experimental uncertainty.

d) Iron. The observation of identifiable lines of highly ionized wall or limiter materials in the tokamak discharge is considerably more difficult than that of the added noble gases, partly because of less control over the amounts, and partly because of higher relevant ionization potentials and the consequent critical balance between the required temperature and the amount of the heavy element. One characteristic of the iron (and perhaps also of molybdenum) concentration is that it generally does not appear to remain a constant fraction of the discharge like krypton, or oxygen, but tends to vary, usually increase, slowly in time. This behavior is probably due to different release mechanism, (evaporation or sputtering rather than desorption or direct recycling), and not different confinement properties of these atoms. 
The observed iron resonance line intensities in a discharge where the total iron concentration appears to rise somewhat more rapidly than usual, are shown in Fig. 6, together with a Mo XIII line. Compared with the discharges discussed above, the limiter radius was increased from 12 to $13 \mathrm{~cm}$, i.e.. closer to the stainless steel vacuum vessel radius, the initial hydrogen density was slightly less, and the heating. current was increased to a peak of 100 kamps (at about $60-80 \mathrm{msec}$ ). The electron temperature was not monitored in detail, but comparison with similar conditions in other occasions suggests that the central temperature rose to somewhat above $2 \mathrm{keV}$ at about $25 \mathrm{msec}$ and dropped. slowly thereafter to the $1.7-1.8$ values shown at $60-80 \mathrm{msec}$, with the radial profile flattened to perhaps $6-8 \mathrm{~cm}$ as indicated on Fig. 1 . The continued increase of the $\mathrm{Fe} X V$ line after $30 \mathrm{msec}$ (which line at that time originates in an approximately cylindrical shell at a radial location where $\mathrm{T}_{\mathrm{e}}(\mathrm{r}) \approx 300 \mathrm{eV}$ ), is an indication of iron influx rate, rather than of density directly. However, if the ion confinement time remains approximately constant in time, it is also a rough measure of total iron density increase in time. [Note that the concurrent change of electron density does not substantially affect the intensity in the she1l, since both the local excitation and ionization rates are proportional to the electron density. See Ref, 11 for a detailed discussion of the radiation emissions.]

The strongest late-appearing line at $\lambda 133.2 \AA$ is ascribed to the $2 s^{2} 1_{s}-2 s 2 p{ }^{1} p$ transition of Fe XXIII, and the $\lambda 192 \AA$ line to $2 s^{2} \mathrm{~S}-2 \mathrm{p}^{2} \mathrm{P}_{3 / 2}$ transition of Fe XXIV. Another line at about 
$256 \AA$ with similar time-behavior and approximately one half the intensity of $\lambda 192 \AA$ line must be the second component of the doublet. In the latter two, and especially the $\lambda 256 \AA$ case the accuracy is only about $\pm 1 \AA$ because of the difficulties of distinguishing the relatively small and slowly varying intensities from the background radiation. All three of these lines have been identified in solar flare spectra. ${ }^{18-21}$

The approximate expected rate coefficients for excitation at $1.8 \mathrm{keV}$ are 5.0 and $3.7 \times 10^{-10} \mathrm{~cm}^{3} / \mathrm{sec}$ for the $\lambda .133 \AA$ and $\lambda 192 \AA$ lines are in reasonable agreement with the observed relative intensities. Comparison with the $\lambda 284 \AA$ line, and its expected rate coefficient, $1 \times 10^{-8} \mathrm{~cm}^{3} / \mathrm{sec}$ at $300 \mathrm{ev}$, and taking into account the increased electron density, indicates an increase of about a factor 3 in iron concentration from $8 \mathrm{msec}$ to $70 \mathrm{msec}$. This is consistent with the observed increase of the $\lambda 284 \AA$ line intensity in time, but detailed measurements of spatial distributions of the light are essential for quantitative conclusions on the ion accumulation rate.

e) Molybdenum. The results of a discharge that favors molybdenum rather than iron lines are shown in Fig. 7. Here the limiter radius was again $12 \mathrm{~cm}$, the initial hydrogen pressure was lower, and the discharge contained relatively more oxygen than in the preceding case. The relative intensities of $\lambda 341 \AA$ Mo XIII (peak at $1.4 \times 10^{15} / \mathrm{cm}^{2}$-sec-sr) and $\lambda 284 \AA \mathrm{Fe} \mathrm{XV}$ (not shown) are approximately reversed compared to Fig. 6. The strongest late-appearing lines, $\lambda 117 \AA$ and $\lambda 129 \AA$ are ascribed to $3 s^{2}{ }^{1} s-3 s 3 p{ }^{1} p$ transition of Mo $\operatorname{xxxI}\left(E_{i}=1805 \mathrm{eV}\right)$, and $3 \mathrm{~s}^{2} \mathrm{~s}-3 \mathrm{p}{ }^{2} \mathrm{P}_{3 / 2}$ of Mo $\operatorname{xxxII}\left(E_{i}=1896 \mathrm{eV}\right)$. The other line of 
the doublet appears to be at $177 \AA$, but the observation is somewhat uncertain because of relatively strong oxygen lines in the vicinity.

The times of appearance and the relative intensities of these lines are consistent with this identification, and so is the relationship to the Fe $\operatorname{XXIII~}\left(E_{i}=1930 \mathrm{eV}\right)$ line. The intensity relationship of the $\lambda 117 \AA$ line to the $\lambda 341 \AA$ Mo XIII line is about the same, taking into account the different electron density change as in the corresponding iron case $(\lambda 284 \AA$ vs. $\lambda 133 \AA$ in Fig. 6). This might be expected on the basis of the estimated excitation rate coefficients $\left(2 \times 10^{-8} \mathrm{~cm}^{3} \cdot / \mathrm{sec}\right.$ for $\lambda 341 \AA$ at $300 \mathrm{eV}, 1.2 \times 10^{-9}$ for $\lambda 117 \AA$ at $\left.2 \mathrm{keV}\right)$.

The principal reason for questioning the identification is the appearance of a strong feature in the solar flare spectrum at $117 \AA$ ascribed ${ }^{20}$ mainly to the ${ }^{2} \mathrm{P}_{1 / 2}-{ }^{2} \mathrm{~S}_{1 / 2}$ resonance line of $\mathrm{Fe} \operatorname{XXII}\left(E_{i}=1794 \mathrm{eV}\right)$. Since iron is definitely present in the tokamak discharge, and the appearance time for the Fe XXII line should be very similar to the observations, the distinction can be made only on the basis of intensity. The beryllium and lithium-like spectra of iron seem to be relatively stronger in the tokamak discharge than in the flare spectrum ${ }^{20}$ - none of the other lines of Fe XIX - XXII could be clearly distinguished from the background. Therefore the observed relative strength of $\lambda 117 \AA$ to $\lambda 133 \AA$ lines can hardly be explained by ascribing the former to Fe XXII ralone. Furthermore, in different experiments such as those shown in Figs. 6 and 7, the $\lambda 117 \AA$ line appears to correlate with molybdenum, rather than iron concentrations. 
Both $\lambda 117 \AA$ A and $\lambda 129 \AA$ Iines have been observed subsequently in the TFR tokamak, ${ }^{11}$ but because of the basic similarity of the devices, the identification problems are also similar. Perhaps stronger support comes from recent Hartree-Fock calculations ${ }^{16}$ that show good agreement with our wavelengths. On the balance we think the identifications to be fairly solidly based. of course, this does not negate the existence of the Fe XXII line, but asserts its contribution to the measured intensity in the tokamak discharge to be minor.

Other molybdenum lines; of earlier states of ionization, that have been measured in ST tokamak discharges are shown in Fig. 8. The identification of the three strongest lines is fairly certain, as they require only a short extrapolation from known sequences, and their time behavior and relative intensities are as expected. The transitions of the weaker lines are unidentified; the ionization states are ascribed on the basis of time of appearance.

\section{SUMMARY}

The wavelengtho and identifications of the obsorved lines are given in Table $I$. The iron and argon lines have already been identified in the solar flares, $17-21$ and some of the molybdenum lines in tokamak discharges have been reported before. 2 The identifications are based on a) changing the plasma composition to determine the element, b) comparing the observed time behavior of the intensity with approximate calculations based on known or estimated rate coefficients at the appropriate electron temperatures and densities, and c) comparison with extrapolated 
isoelectronic sequences. In most cases there are also recent ab initio calculations available. for comparison. (Because of some possibilities of interfering lines of $\mathrm{Fe}, \mathrm{Cr}$ or $\mathrm{Ni}$ ions at states of similar ionization potential, the identification of the Mo XXXI and XXXII lines should perhaps be regarded as tentative until some independent confirmation.) The measured wavelengths are accurate to $\pm 0.5 \AA$ in the cases where the decimal is shown and to $\pm 1 \stackrel{\circ}{\AA}$ where it is not. The measured intensities have given useful information about relative intensities of doublets, and also of lines of adjacent. states of ionization. In the case of added noble gases, the known amounts and measured intensities allow the deduction of rate coefficients for excitation, which in turn can be used to evaluate the amounts of spontaneously occurring elements from measured intensities of analogous transitions.

The accuracy of such deductions of concentration is adequate to establish the order of magnitude of the various plasma constituents and estimate their overall influence on the plasma behavior, e.g. their probable contributions to electrical resistivity and local energy loss by radiation. However, it is not satisfactory for finer detail, such as the important problem of the accumulation rate of heavy ions in the discharge. Improvement in this situation must be sought in two directions: experimentally by providing radial distributions of the line emissivities, and more detailed and accurate intensity measurement calibrations, and theoretically by providing transition probabilities and rate coefficients for excitation of the 
appropriate lines, and of ionization and recombination of the ions that can be relied on at least to 20-30z.

\section{ACKNOWLEDGMENTS}

The wavelength and intensity measurements reported here were carried out during various plasma physics experiments on the ST Tokamak in collaboration with Dr. E. B. Meservey, Dr. D. Dimock, Dr. L. C. Johnson, Dr. N. Bretz, and other members of the ST group.

This work was supported by U. S. Energy Research and Development Administration Contract E(11-1)-3073. 


\section{REFERENCES}

1. H. P. Furth, Nucl. Fusion 15, 487 (1975).

2. D. L. 'Dimock et al., Plasma Physics and Controlled Nuclear Fusion Research (Proc. 4th Int. Conf., Madison, 1971) 1 , IAEA (1971) 451.

3. D. L. Dimock et al., Nucl. Fusion 13, 271 (1973).

4. S. von Goeler et al., Nucl. Fusion 15, 301 (1975).

5. E. Hinnov and F. W. Hofmann, J. Opt. Soc. Am. 53, 1259 (1963).

6. J. G. Hirschberg and E. Hinnov, J. Chem. Phys. 45, 2233 (1966).

7. L. C. Johnson and E. Hinnov, J. Quant. Spectrosc. Radiat. Transfer 13,333 (19.73).

8. B. L. Moiseiwitsch and S. J. Smith, Rev. Mod. Phys. 40 , 238 (1968).

9. O. Bely, Proc. Phys. Soc. of London 88, 587 (1966).

10. J. Davis, J. Quant. Spectrosc. Radiat. Transfer 14, 549 (1974).

11. Equipe TFR, Nucl. Fusion 15, 1053 (1975).

12. N. Bretz et al., Nucl. Fusion 15, 313 (1975).

13. B. Edlen, Encyclopedia of Physics (Springer Verlag, Berlin, 1964), S. Flugge, ed., Vol. XXVII, p. 80.

14. A. W. Weiss and W. L. Wiese, Bull. Am. Phys. Soc. II, 20, 1295 (1975).

15. D. E. Parks, private communication.

16. A. W. Weiss, to be published.

17. B. C. Fawcett et al., J. Phys. B 4, 986 (1971).

18. J. D. Purcell and K. G. Widing, Astrophys. J. 176, 239 (1972). 
19. S. O. Kastner, W. M. Neupert, M. Swartz, Astrophys. J. 191,261 (1974).

20. B. C. Fawcett and R. D. Cowan, Mon. Not. R. Astron, Soc. 171,1 (1975).

21. K. G. Widing, Astrophys. J. 197, L33 (1975). 
TABLE I. Wavelengths and Transitions of the Lines Observed in the ST Tokamak.

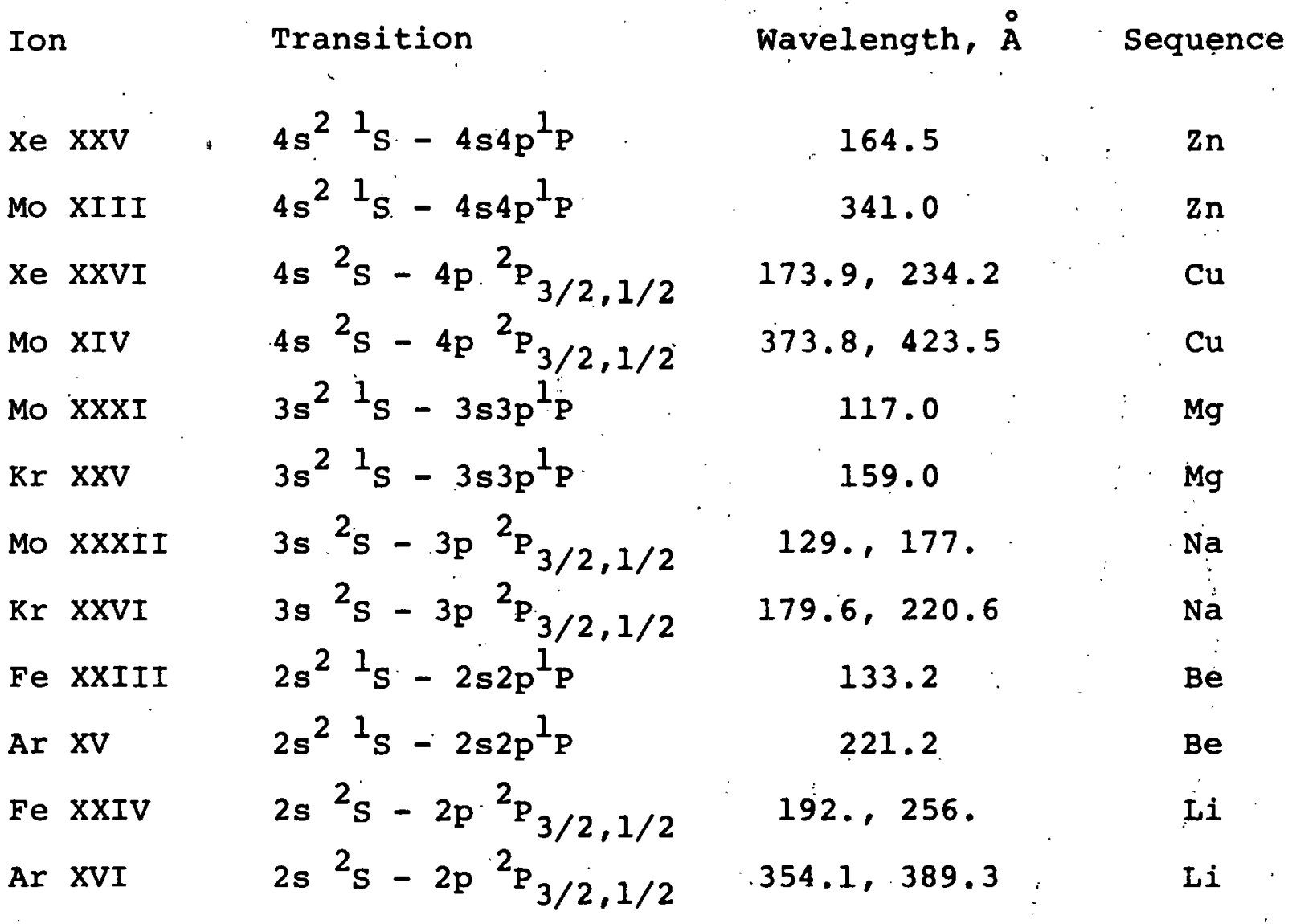




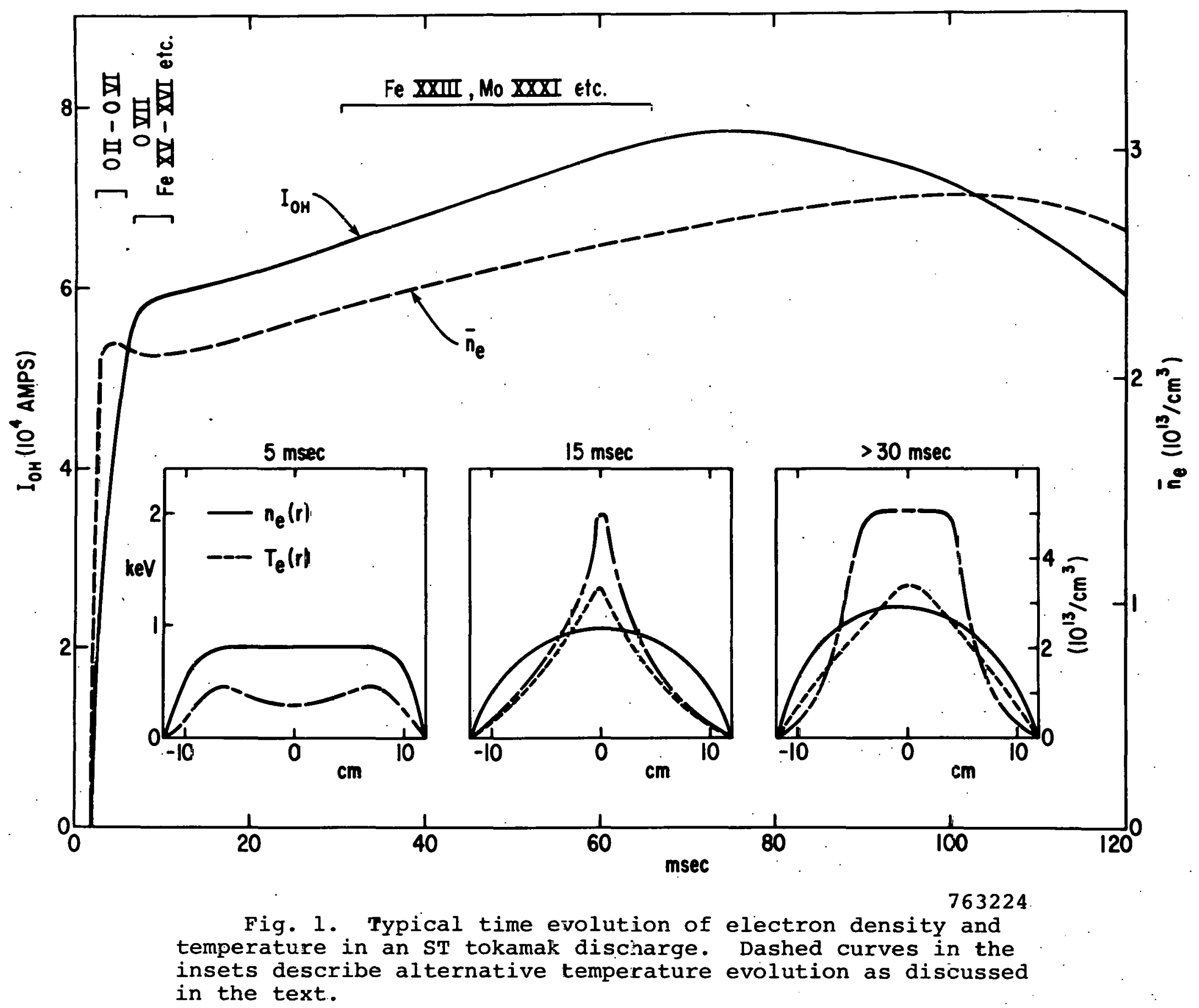




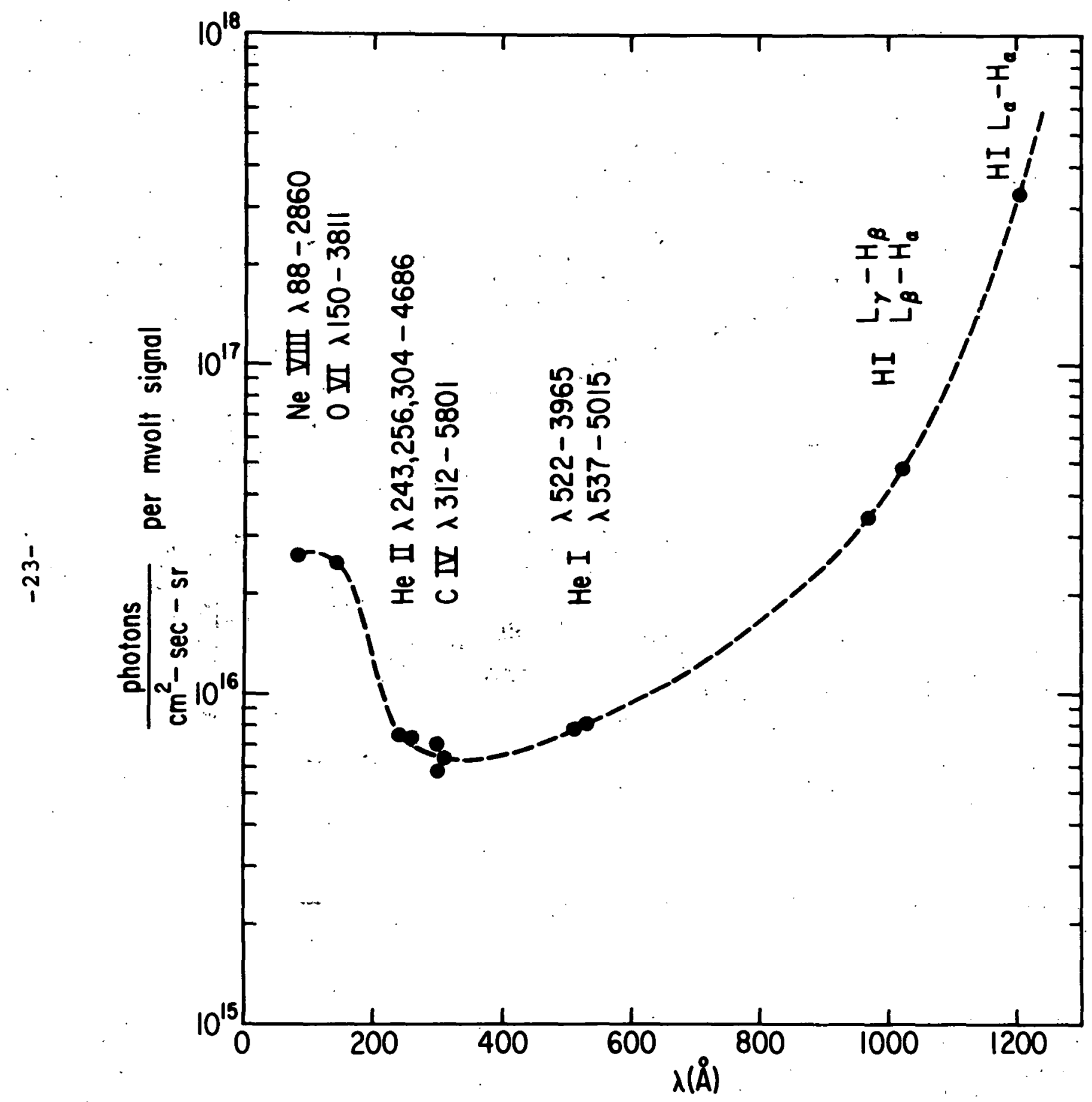

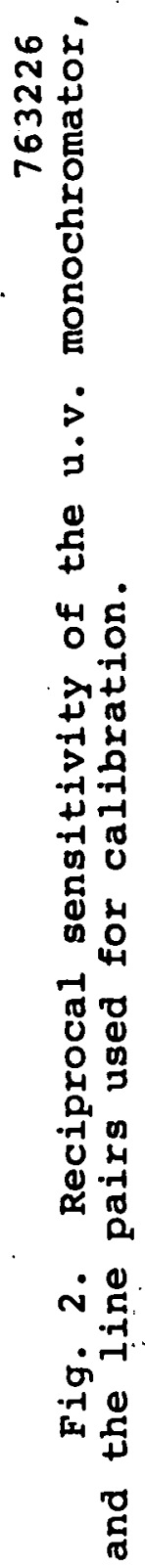




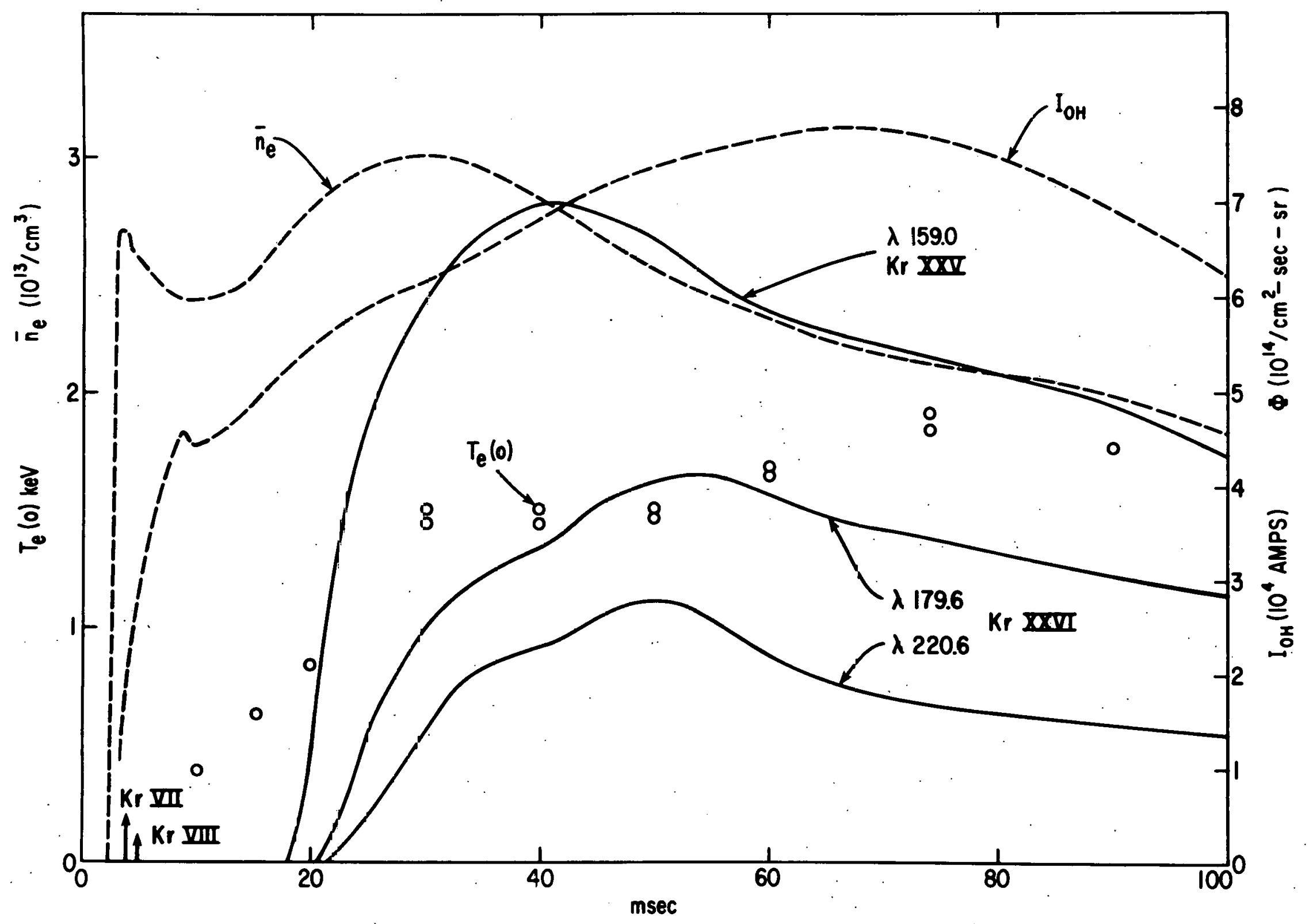

Fig. 3. The heating current, average electron density, central electron temperature (o) and the intensities of $\mathrm{Mg}$ and $\mathrm{Na}$ sequence krypton resonance lines vs time. The arrows indicate time of appearance of $\mathrm{Zn}$ and $\mathrm{Cu}$ sequence krypton lines shown in Fig. 4. 


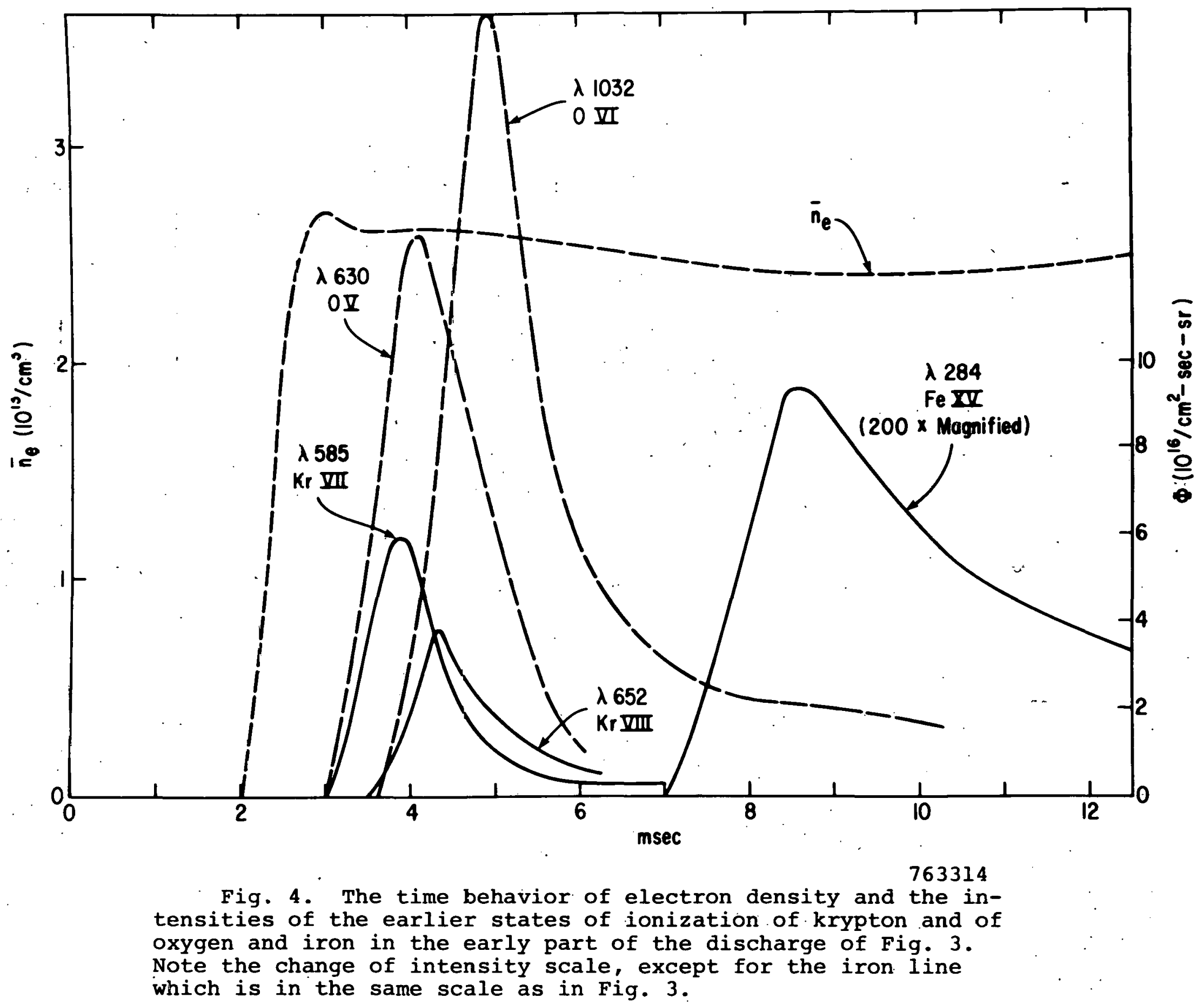




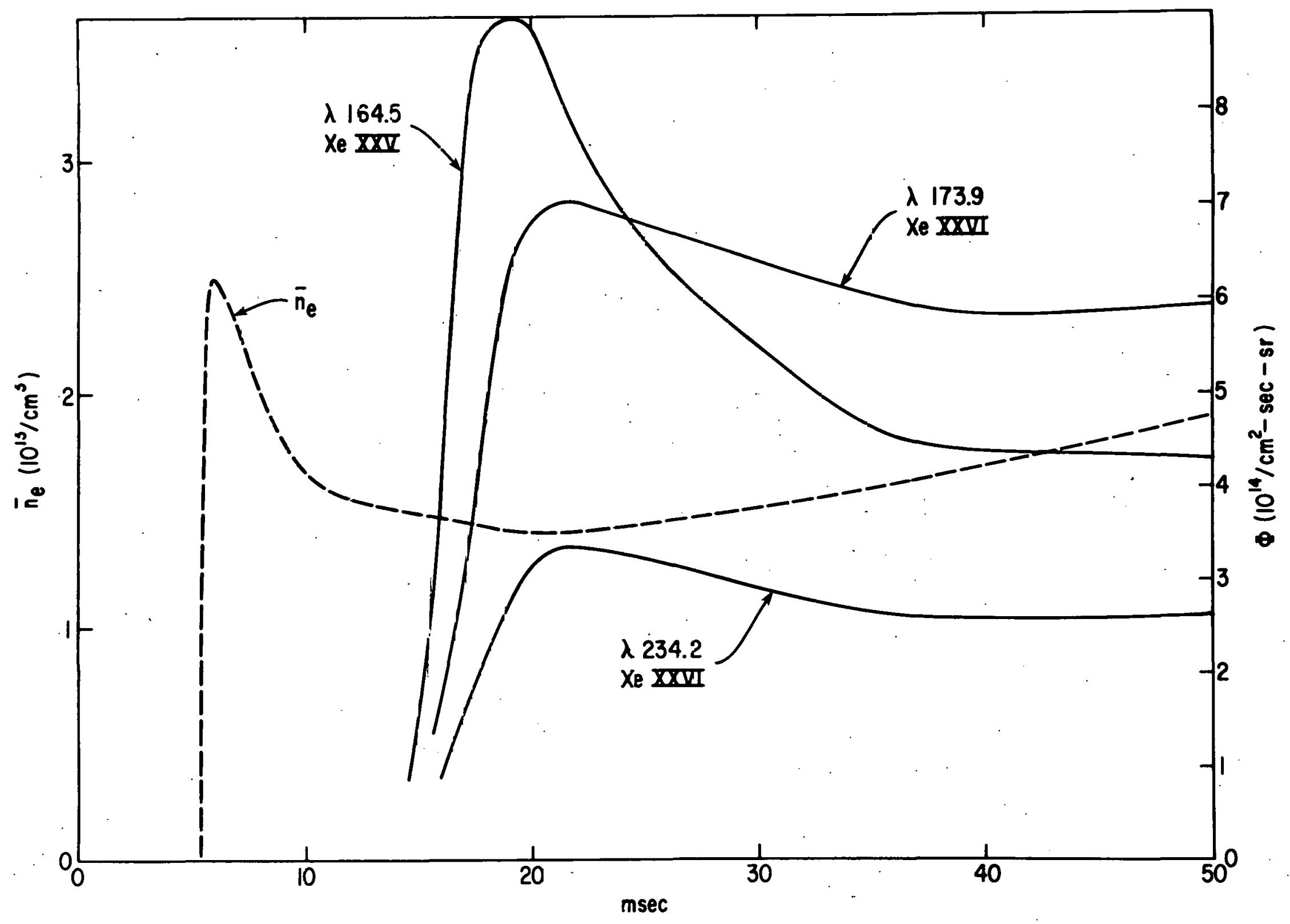
lines of xenon ard the average electron density. 


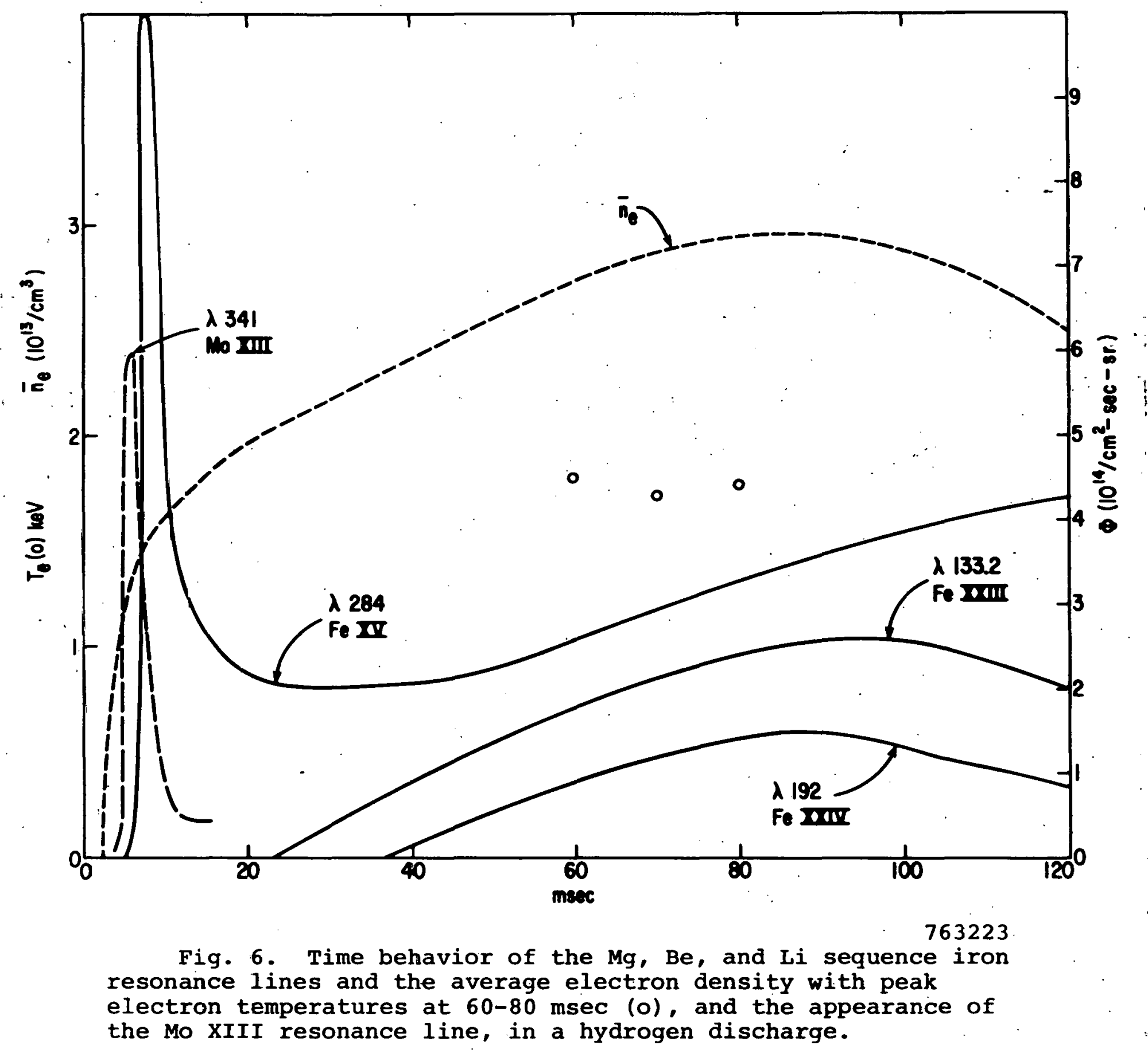




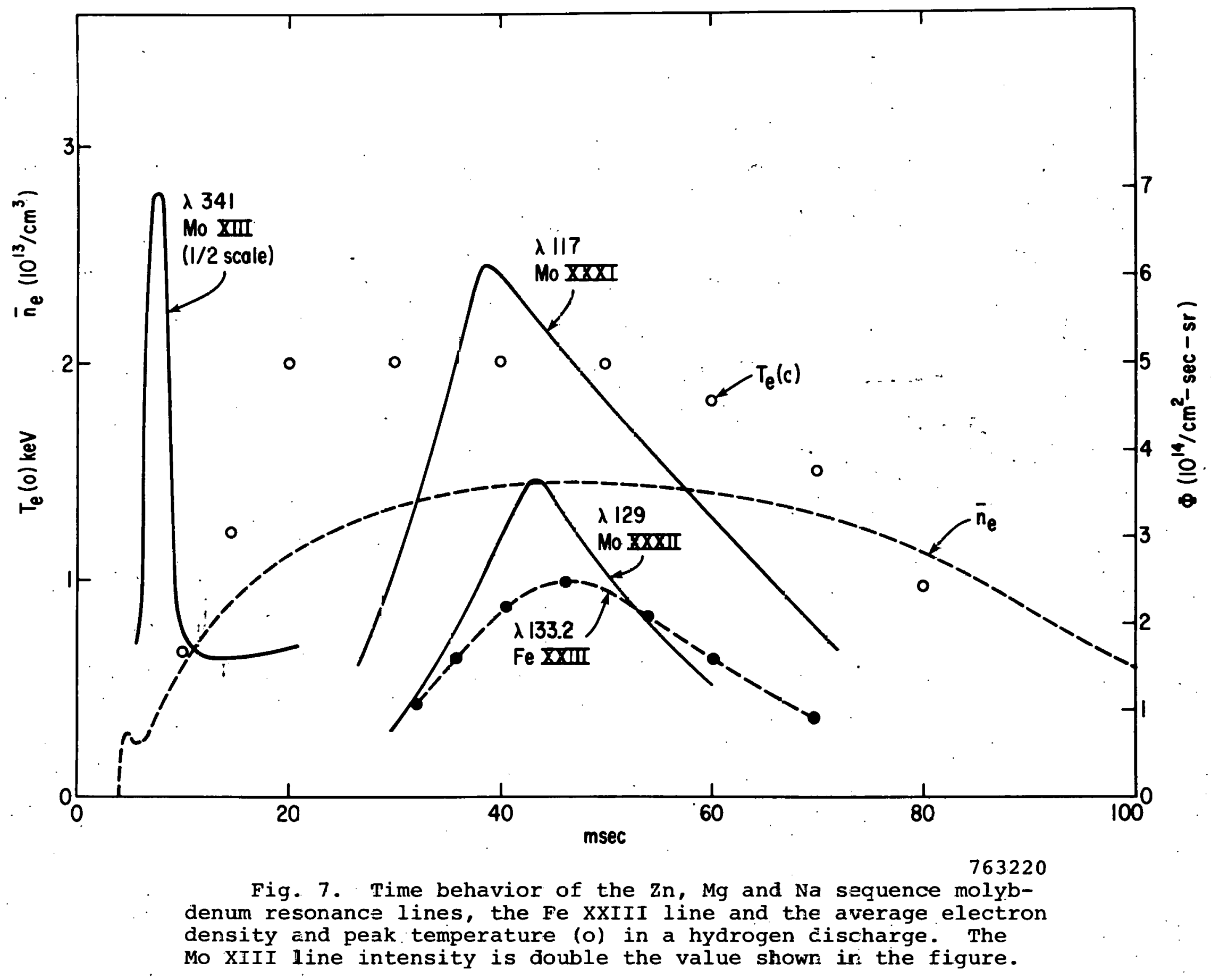


RELATIVE INTENSITY

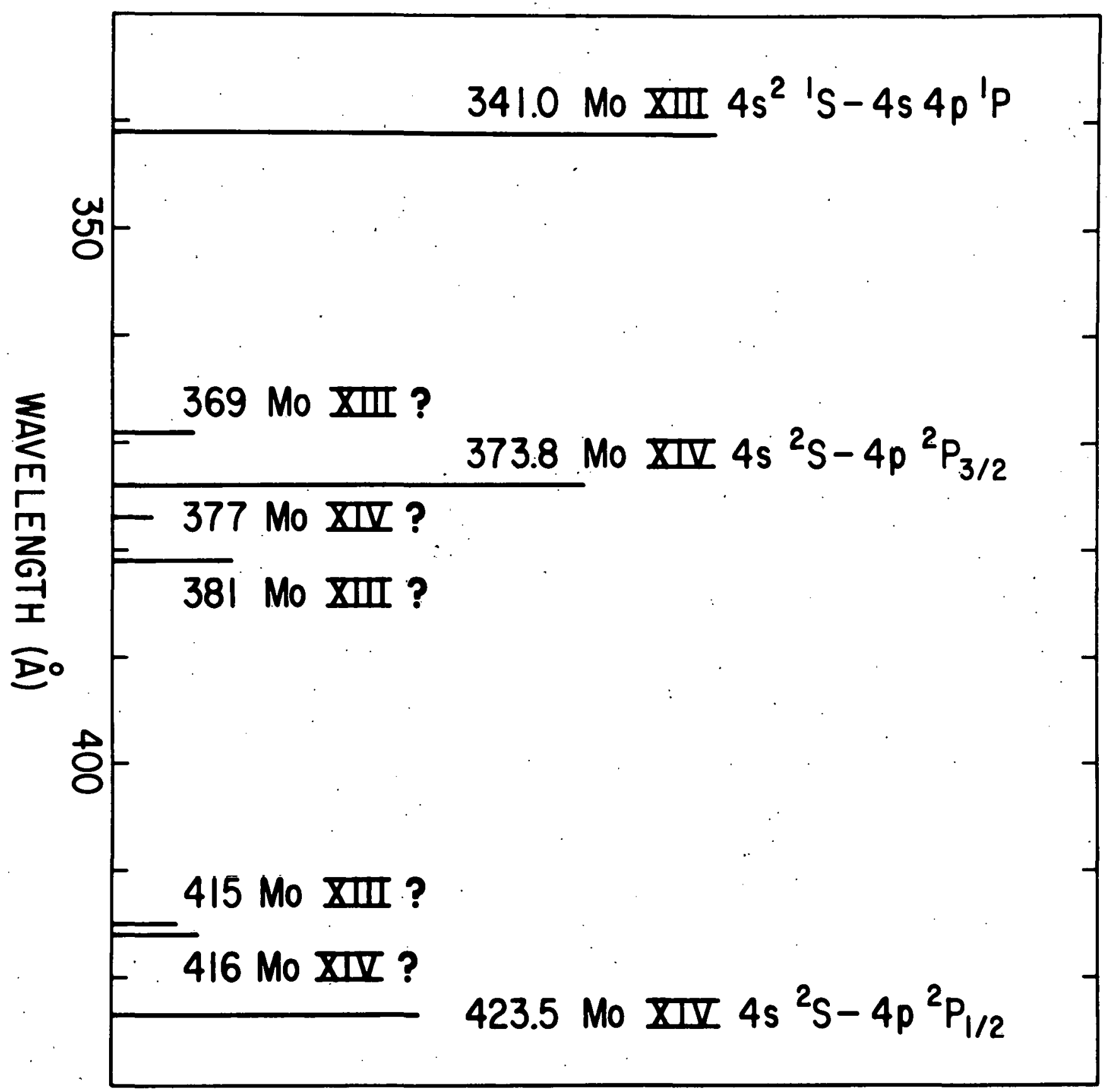

763225

Fig. 8. Wavelengths and relative intensities of several Mo XIII and XIV lines. 